\title{
Complex surface diffusion mechanisms of cobalt phthalocyanine molecules on $\mathrm{Ag}(100)$
}

\author{
Grażyna Antczak ${ }^{*}$, Wojciech Kamiński ${ }^{\ddagger}$, Agata Sabik ${ }^{\ddagger}$, Christopher Zaum ${ }^{\dagger}$, Karina \\ Morgenstern $^{\S}$ \\ ${ }^{*}$ Institute of Experimental Physics, University of Wrocław, Wrocław, Poland \\ ${ }^{\dagger}$ Leibniz University Hannover, Hannover, Germany \\ ${ }^{\S}$ Chair for Physical Chemistry I, Ruhr-Universität Bochum, Bochum, Germany \\ *Corresponding authore-mail: antczak@ifd.uni.wroc.pl
}

Figure S1 shows the stage of $0.1 \mathrm{ML}$ of CoPc adsorption after annealing the sample to $50 \mathrm{~K}$ and immobilized by cooling down to $5 \mathrm{~K}$. It shows that the islands are rarely arranged by heating the sample, only occasional dimers and trimers are visible.

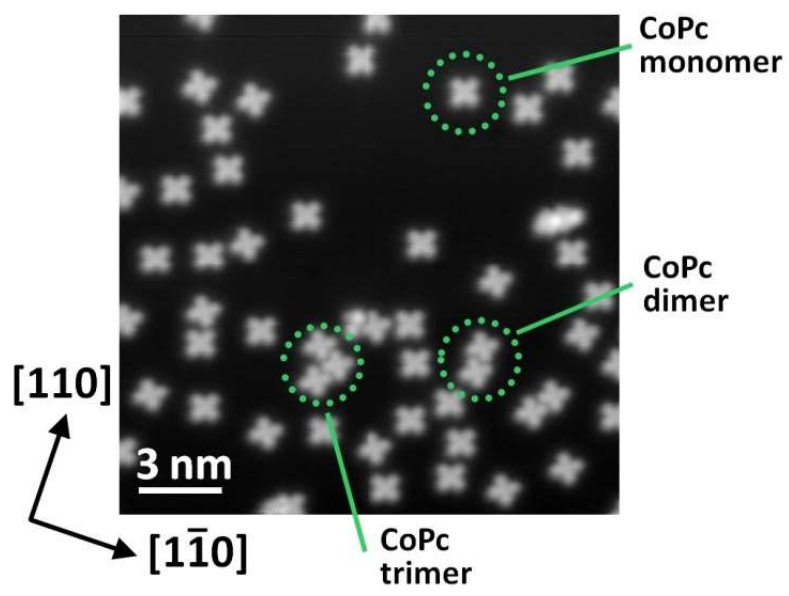

Figure S1. STM image of CoPc molecule adsorbed on $\mathrm{Ag}(100)$ surface after $50 \mathrm{~K}$ annealing imaged at $5 \mathrm{~K}$, a monomer, a dimer and trimer marked by green circles.

Figure S2 presents the positions visited by three CoPc molecules and dimer during heating the sample to $43 \mathrm{~K}$. We can see that molecules as well as dimer making discrete jumps along $<110>$ directions. 


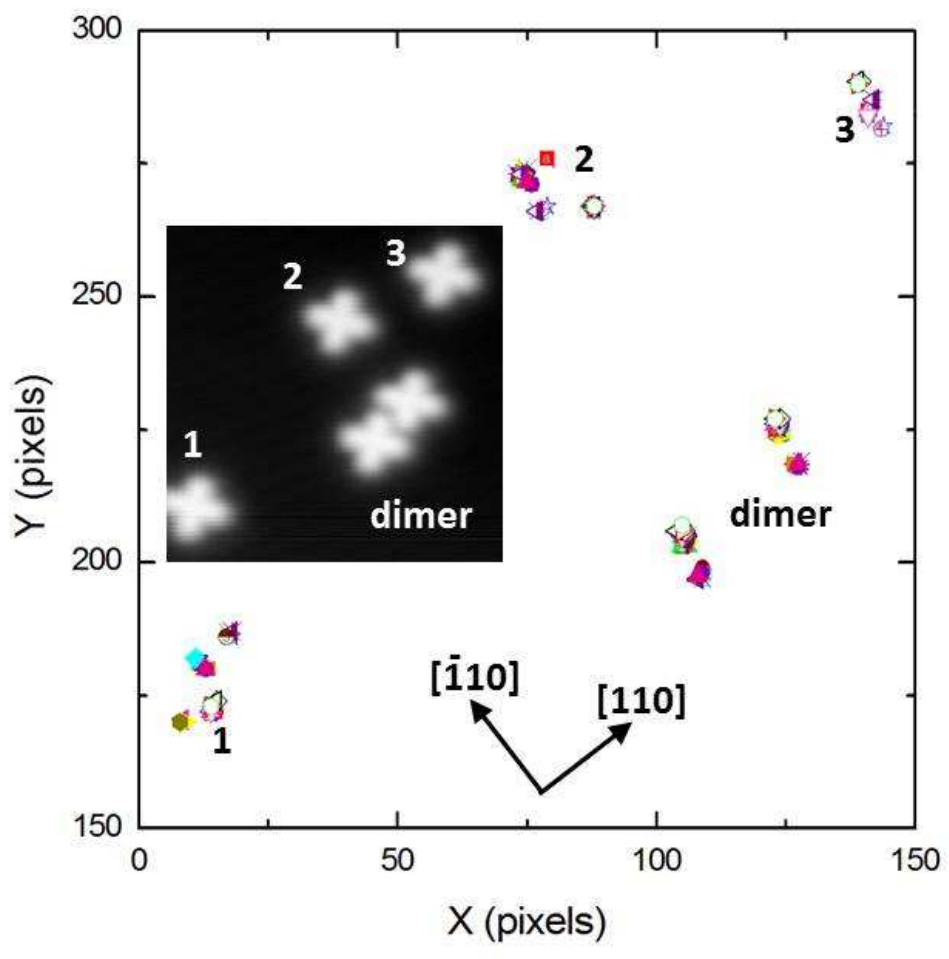

Figure S2. The adsorption places visited during 45 images by three molecules (denoted as 1, 2 and 3 ) and dimer during motion at $43 \mathrm{~K}$. Different colors and shapes of points are associated with positions of the molecules on different STM images.

The movie provided in the Supporting Information shows the thermal diffusion of CoPc molecules at $49 \mathrm{~K}$. The movie consists 83 images and runs over time of $16185 \mathrm{~s}$. 